\title{
Bioactive peptide from Pyropia yezoensis and its anti-inflammatory activities
}

\author{
HYUN-AH LEE, IN-HYE KIM and TAEK-JEONG NAM \\ Institute of Fisheries Science, Pukyong National University, Busan 619-911, Republic of Korea
}

Received August 10, 2015; Accepted October 12, 2015

DOI: $10.3892 / \mathrm{ijmm} .2015 .2386$

\begin{abstract}
Pyropia yezoensis (P. yezoensis) is an important marine algae. Its high protein content serves as a good source of biologically active peptides. Potent inhibitory effects on the production of inflammatory mediators were observed in a bioactive peptide derived from $P$. yezoensis (peptide from $P$. yezoensis; PPY1), as demonstrated in lipopolysaccharide (LPS)-stimulated macrophages. The present study showed that peptide concentrations ranging from 250 to $1,000 \mathrm{ng} / \mathrm{ml}$ had no significant cytotoxicity in the cell viability assay when applied to the RAW 264.7 cells for $24 \mathrm{~h}$. PPY1 completely inhibited LPS-stimulated nitric oxide (NO) release in a dose-dependent manner. Fluorescence intensity, corresponding to intracellular reactive oxygen species (ROS) produced by $10 \mathrm{ng} / \mathrm{ml}$ LPS-stimulated cells, significantly shifted, indicating that the peptide reduced the level of ROS. Furthermore, PPY1 exerted potent inhibitory activity to reduce the release of pro-inflammatory cytokines (inducible NO synthase, cyclooxygenase-2, interleukin-1 $\beta$ and tumor necrosis factor- $\alpha$ ) in LPS-stimulated macrophages in a dose-dependent manner. These results also showed that the anti-inflammatory activity of PPY1 was associated with downregulation of extracellular signal-regulated kinase, protein 38 , and c-jun $\mathrm{NH}_{2}$-terminal kinase phosphorylation in the mitogen-activated protein kinase pathways. In conclusion, PPY1 can have a significant role as an anti-inflammatory agent, with a potential for use in marine products.
\end{abstract}

\section{Introduction}

Pyropia yezoensis (P. yezoensis) is an important marine algae that is cultivated primarily in China, Japan and Korea (1). It is exposed to adverse environmental conditions, such as

Correspondence to: Professor Taek-Jeong Nam, Institute of Fisheries Sciences, Pukyong National University, 474 Ilgwang-ro, Ilgwang, Gijang, Busan 619-911, Republic of Korea

E-mail: namtj@pknu.ac.kr

Key words: Pyropia yezoensis, bioactive peptide, anti-inflammation, RAW 264.7 high light intensity and oxygen concentration, which lead to the formation of free radicals and other strong oxidizing agents without causing serious photodynamic damage (2-4). Consequently, $P$. yezoensis may be capable of generating the necessary compounds to protect itself from external factors, such as pollution, stress and ultraviolet (UV) radiation.

There are various biologically active phytochemicals in $P$. yezoensis, including carotenoids, fatty acids, polysaccharides, proteins, vitamins, tocopherol and phytocyanins $(5,6)$, which have shown various medicinal effects $(7,8)$. In particular, its high protein content makes $P$. yezoensis a potential source of bioactive peptides and an alternative to synthetic drugs, with antihypertensive (8), anti-inflammatory $(9,10)$, antioxidant (11-13), anticancer (14) and tissue-healing properties (15-17).

Bioactive peptides usually contain 3-20 amino acid residues, and their activities are based on their amino acid composition and sequence (18). These short amino acid chains are inactive within the sequence of the parent protein but can be released during gastrointestinal digestion, food processing or fermentation. Marine-derived bioactive peptides have been obtained through enzymatic hydrolysis and have been shown to drive numerous fundamental metabolic processes (19-21).

Inflammation is an effective immune response to foreign stimuli and ultimately results in restoration of normal function. Macrophages are important mediators of the inflammatory response (22). Activated macrophages produce pro-inflammatory enzymes, such as inducible nitric oxide synthase (iNOS), cyclooxygenase-2 (COX-2), and various pro-inflammatory cytokines, such as tumor necrosis factor- $\alpha$ (TNF- $\alpha$ ) and interleukins (IL- $1 \beta$ and IL-6), which serve as essential mediators of the inflammatory response (23). However, excessive and uncontrolled production of these inflammatory mediators and cytokines are associated with autoimmune disorders, neuropathological diseases, rheumatoid arthritis, microcirculatory dysfunction and tissue damage, leading to fatality. Suppression of these inflammatory mediators may be an effective therapeutic strategy to prevent diseases caused by inflammatory disorders (24).

Mitogen-activated protein kinases (MAPKs) are a family of serine/threonine protein kinases that mediate fundamental biological processes and cellular responses to external stress signals. Increased activity of MAPK and their involvement in regulating the synthesis of inflammation mediators at the levels of transcription and translation makes them potential targets for anti-inflammatory therapeutics (25). Modulation of the 
activity of the protein 38 (p38), extracellular signal-regulated kinase (ERK), and c-jun $\mathrm{NH}_{2}$-terminal kinase (JNK) signaling cascades on the MAPK pathways is regarded as an attractive strategy, as they are capable of reducing the synthesis of pro-inflammatory cytokines and their signaling.

The increasing incidence of inflammation has led to the search for proteins and peptides, and their anti-inflammatory effects have been identified in numerous studies. According to Zhu et al (26), TC26RFa from the tree shrew of Tupaia belangeri chinensis may inhibit inflammatory factor secretion induced by lipopolysaccharides (LPS). The inhibition of lunasin on pro-inflammatory cytokines via suppressing the nuclear factor- $\kappa \mathrm{B}$ pathway has also been suggested (22). The egg white peptide was a promising novel therapeutic approach to treating the inflammatory bowel disease by reducing the local expression of pro-inflammatory cytokines (27). However, limited research on the production of anti-inflammatory peptides from $P$. yezoensis has been reported. The present study investigated the anti-inflammatory properties of PPY1 in LPS-stimulated Raw 264.7 cells as an in vitro model and assessed the anti-inflammatory mechanism of PPY1 with the potential contribution to MAPK signaling.

\section{Materials and methods}

Preparation of peptide. The peptide PPY1, derived from $P$. yezoensis, was synthesized by Peptron Inc. (Daejeon, Korea) and the sequence was K-A-Q-A-D. Purification of PPY1 was performed using the Shimadzu Prominence HPLC apparatus and controlled using the software package Class-VP, 6.14 (Shimadzu Corp., Kyoto, Japan). A C18 column (Shiseido Capcell Pak; Shiseido, Tokyo, Japan) in $0.1 \%$ trifluoroacetic acid (TFA)/water and a gradient of $10-70 \%$ acetonitrile in $0.1 \%$ TFA, with a flow rate of $1 \mathrm{~nm} / \mathrm{min}$ and $\mathrm{UV}$ detection at $220 \mathrm{~nm}$, was used. The molecular weight of PPY1 was determined to be 532 Da (Fig. 1) using mass spectrometer analysis (HP 1100 series LC/MSD; Agilent Technologies, Inc., Santa Clara, CA, USA).

Cell culture. The mouse macrophage cell line RAW 264.7 was obtained from the American Type Culture Collection (Rockville, MD, USA) and cultured in Dulbecco's modified Eagle's medium supplemented with $10 \%$ fetal bovine serum, $50 \mu \mathrm{g} / \mathrm{ml}$ penicillin, $25 \mu \mathrm{g} / \mathrm{ml}$ amphotericin $\mathrm{B}$, and $50 \mu \mathrm{g} / \mathrm{ml}$ streptomycin at $37^{\circ} \mathrm{C}$ in a humidified atmosphere containing $5 \% \mathrm{CO}_{2}$.

Cell proliferation assay. RAW 264.7 cell proliferation was evaluated using the Cytox ${ }^{\mathrm{TM}}$ cell viability assay kit (LPS solution, Daejeon, Korea). Cells were plated at a density of $1 \times 10^{4}$ cells/well in 96-well plates overnight and subsequently treated with PPY1 (250, 500 and 1,000 ng/ml) for $24 \mathrm{~h}$. At the end of the treatment period, $10 \mu \mathrm{l}$ of the cell viability assay solution was added to each well, and the plate was incubated for $30 \mathrm{~min}$. The absorbance at $450 \mathrm{~nm}$ was measured using a microplate reader (BioTek Instruments, Inc., Winooski, VT, USA). The results are expressed as a percentage of the control.

Nitric oxide (NO) measurement. Accumulated nitrite $\left(\mathrm{NO}_{2}^{-}\right)$ in the culture media was measured following a modified version of the assay described by Green et al (28) RAW 264.7 cells were plated at a density of $1 \times 10^{4}$ cells/well on a 96-well plate and incubated overnight. Subsequently, cells were stimulated with LPS (10 ng/ml) and treated with PPY1 (250, 500 and $1,000 \mathrm{ng} / \mathrm{ml})$. A volume of $100 \mu \mathrm{l}$ of the cultured supernatant was plated in a 96-well plate, and $100 \mu \mathrm{l}$ of Griess reagent was added. The plate was incubated for $10 \mathrm{~min}$ and the absorbance at $540 \mathrm{~nm}$ was measured using a microplate reader.

Determination of intracellular reactive oxygen species (ROS). The intracellular ROS were detected using the ROS-sensitive fluorescent dye, CM-H2DCFDA. Cells were plated at $1 \times 10^{4}$ cells/well in 96-well plates overnight and were subsequently treated with PPY1 $(250,500$ and 1,000 ng/ml) for $24 \mathrm{~h}$. Cells were stimulated with LPS $(10 \mathrm{ng} / \mathrm{ml})$ during the last $18 \mathrm{~h}$ of treatment with peptides. At the end of the treatment, cells were washed with cold phosphate-buffered saline (PBS) and incubated with $10 \mu \mathrm{M} \mathrm{CM}-\mathrm{H} 2 \mathrm{DCFDA}$ at $37^{\circ} \mathrm{C}$ for $30 \mathrm{~min}$. Following incubation, the cells were washed with cold-PBS and the fluorescence intensities of the stained cells were determined in a FilterMax F5 microplate reader (Molecular Devices LLC, Sunnyvale, CA, USA) using excitation and emission wavelengths of 485 and $535 \mathrm{~nm}$, respectively.

Western blot analysis. The whole cell extracts for immunoblotting iNOS, COX-2, TNF- $\alpha$, IL-1 $\beta$ and MAPKs were isolated from LPS-stimulated RAW 264.7 cells. A protein sample $(35 \mu \mathrm{g})$ was loaded in SDS-PAGE and transferred to a PVDF membrane (Millipore Corp., Billerica, MA, USA). The membrane was blocked with $1 \%$ bovine serum albumin (BSA) in Tris-buffered saline and Tween 20 (TBS-T) buffer and subsequently incubated with primary antibodies [iNOS (sc-650), COX-2 (sc-1745), TNF- $\alpha$ (sc-1350), IL-1 $\beta$ (sc-7884), p-ERK (sc-7383), ERK (sc-94), p-p38 (sc-7973), p38 (sc-7149), p-JNK (sc-6254), JNK (sc-7345) GAPDH (sc-25778) (1:1,000 in $1 \% \mathrm{BSA} / \mathrm{TBS}-\mathrm{T})]$ overnight at $4^{\circ} \mathrm{C}$. The membranes were then washed twice for $15 \mathrm{~min}$ in TBS-T. The secondary antibody was a horseradish peroxidase (HRP)-conjugated goat anti-mouse or rabbit antibody [goat anti-mouse IgG-HRP (sc-2031), goat anti-rat IgG-HRP (sc-2032) (1:10,000 in 1\% BSA/TBS-T)]. Following incubation and repeated washing, the expression of each protein was visualized using chemiluminescence substrate (Advansta, Menlo Park, CA, USA) and visualized using the GeneSys imaging system (SynGene Synoptics, Ltd., London, UK).

Statistical analysis. All the samples were analyzed in triplicate, and the results are expressed as means \pm standard error of the mean. SPSS (SPCC Inc., Chicago, IL, USA) was used to perform the statistical analyses. $\mathrm{P}<0.05$ was considered to indicate a statistically significant difference.

\section{Results}

Cell viability of RAW 264.7 macrophages cells. To determine the effect of PPY1 on cell viability, PPY1 was tested in the Cyto $\mathrm{X}^{\mathrm{TM}}$ cell viability assay using RAW 264.7 macrophage cells. Proliferation and cytotoxic effects were examined to establish the appropriate concentration ranges of PPY1 for analysis in the following experiments (Fig. 2). A range of concentrations (250-1,000 ng/ml) of PPY1 had no cytotoxic effects on RAW 264.7 cells and could be used in further studies. 

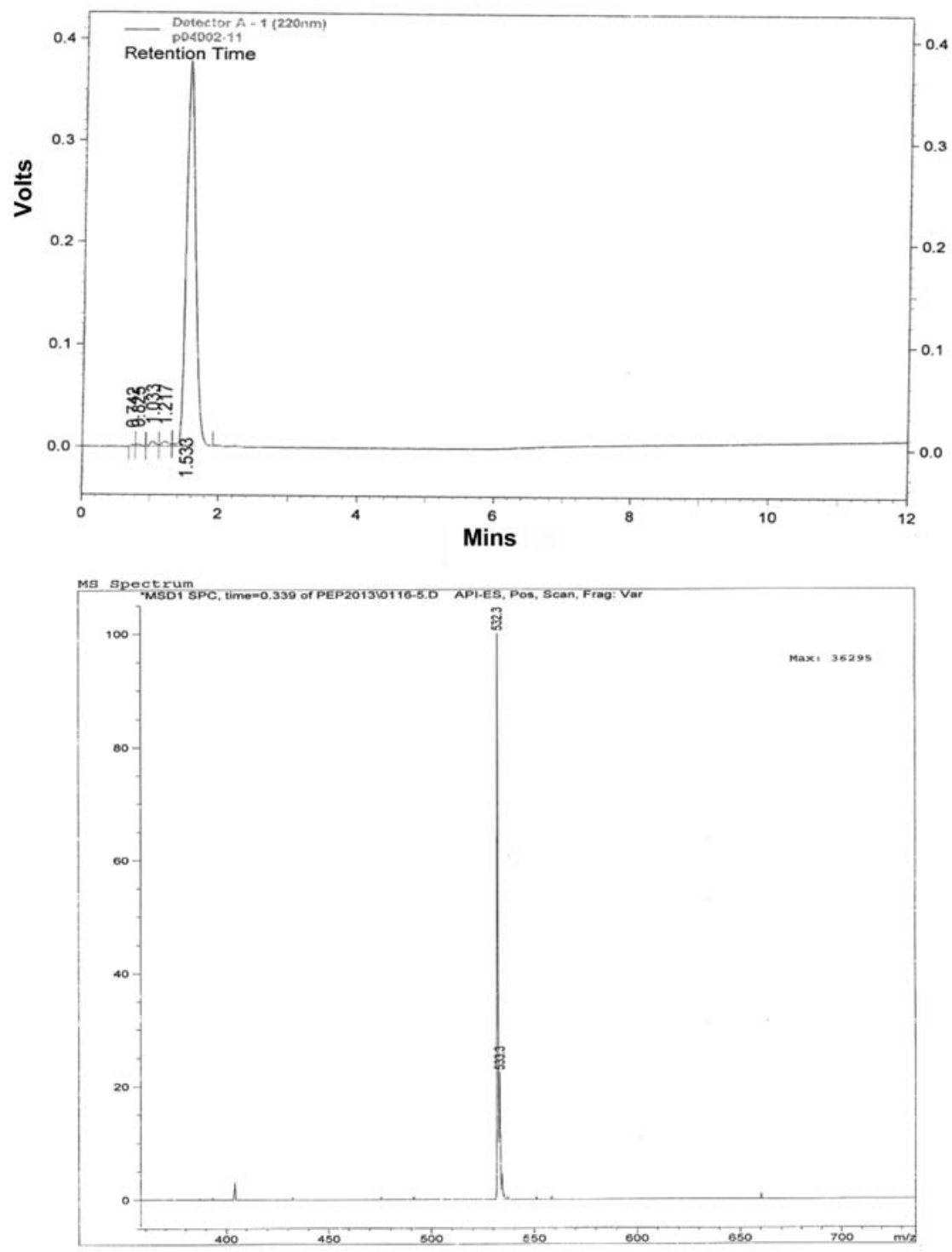

Figure 1. Separation of bioactive peptide from P. yezoensis (PPY1) by high-performance liquid chromatography (Capcell Pak, C18 column) and mass spectrometry analysis (HP 1100 series LC/MSD).
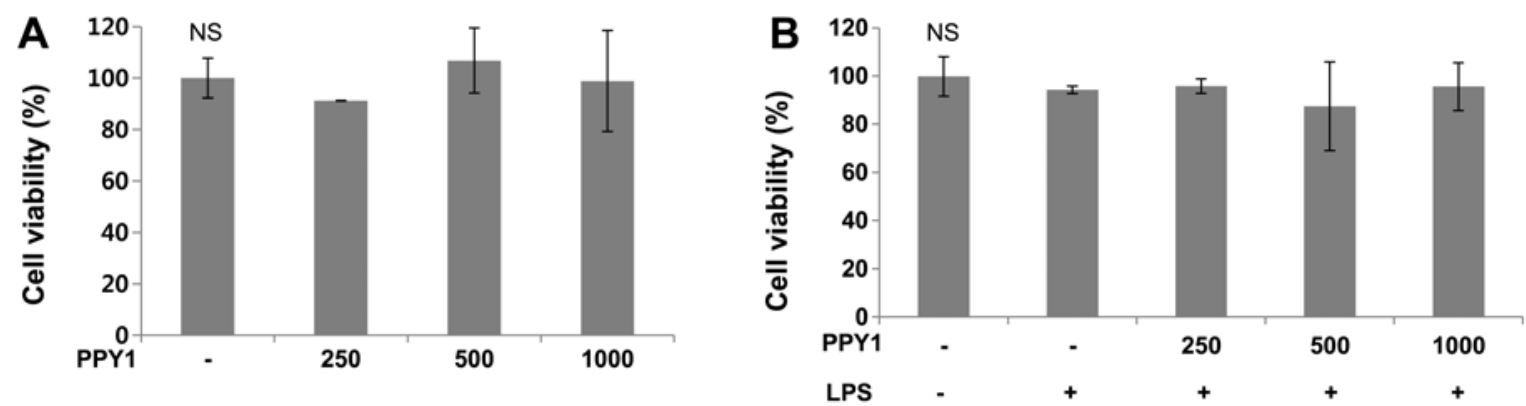

Figure 2. Effect of bioactive peptide from P. yezoensis (PPY1) on the proliferation and cytotoxicity in (A) RAW 264.7 macrophages and (B) LPS-stimulated RAW 264.7 macrophage. (A) RAW 264.7 macrophages were plated at a density of 1x104 cells/well in 96-well plates and treated with PPY1 (250, 500 and 1,000 ng/ml) for $24 \mathrm{~h}$. (B) RAW 264.7 macrophages were stimulated with LPS $(10 \mathrm{ng} / \mathrm{ml})$ and treated with PPY1 $(250,500 \mathrm{and} 1,000 \mathrm{ng} / \mathrm{ml})$. The absorbance at $450 \mathrm{~nm}$ was measured using a microplate reader. The results are expressed as a percentage of the control. Data are presented as the mean \pm standard error of the mean and $\mathrm{P}<0.05$ was considered to indicate a statistically significant difference. LPS, lipopolysaccharide. NS, not significant.

PPY1 inhibits NO and ROS production in LPS-stimulated $R A W 264.7$ macrophage cells. NO has a central role in inflammation, and its level can be quantified by a simple procedure.
RAW 264.7 cells were treated with LPS (10 ng/ml) for $24 \mathrm{~h}$ to examine the inhibitory effect of PPY1 on LPS-stimulated NO production. The LPS treatment significantly $(\mathrm{P}<0.05)$ 

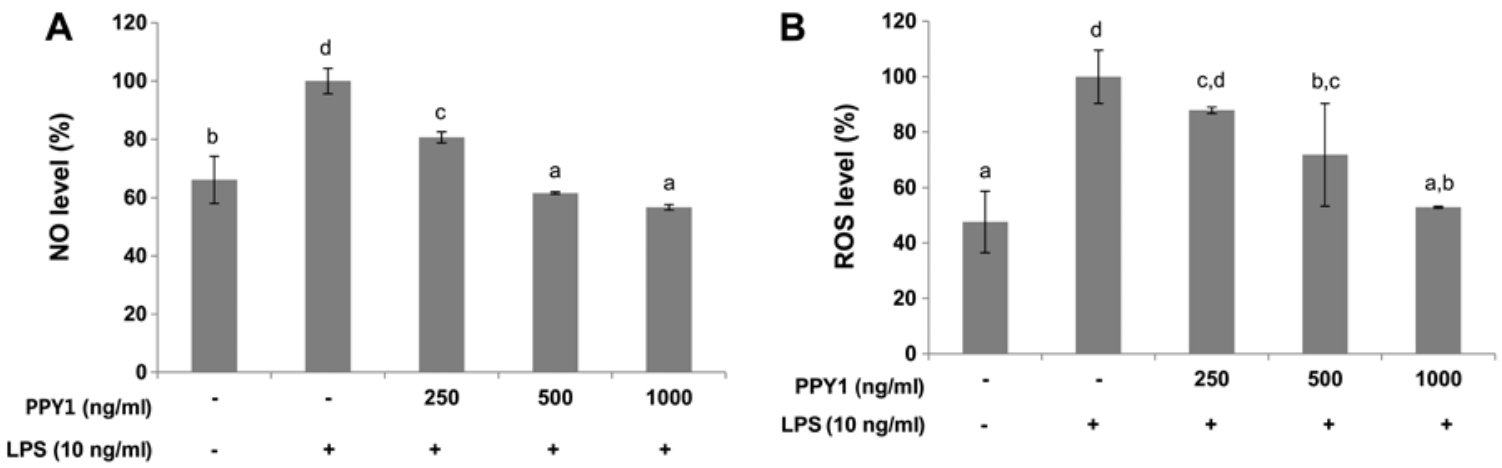

Figure 3. Effect of the bioactive peptide from P. yezoensis (PPY1) on the level of (A) NO and (B) ROS in LPS-stimulated RAW 264.7 macrophages. (A) RAW 264.7 cells were seeded in a 96-well plate and stimulated with LPS (10 ng/ml) and treated with PPY1 (250,500 and 1,000 ng/ml). The absorbance at $540 \mathrm{~nm}$ was measured following the addition of Griess reagent with cultured supernatant. (B) RAW 264.7 cells were seeded in 96-well plates and subsequently treated with PPY1 $(250,500$ and $1,000 \mathrm{ng} / \mathrm{ml})$ for $24 \mathrm{~h}$. Cells were stimulated with LPS $(10 \mathrm{ng} / \mathrm{ml})$ during the last $18 \mathrm{~h}$ of treatment with PPY1. At the end of the treatment, cells were incubated with CM-H2DCFDA and the fluorescence intensities of the stained cells were determined in a FACScan XL flow cytometer using excitation and emission wavelengths of 485 and $535 \mathrm{~nm}$. Data are presented as the mean \pm standard error of the mean. ${ }^{\mathrm{a}-\mathrm{d}} \mathrm{P}<0.05$ was considered to indicate a statistically significant difference. NO, nitric oxide; ROS, reactive oxygen species; LPS, lipopolysaccharide.

A

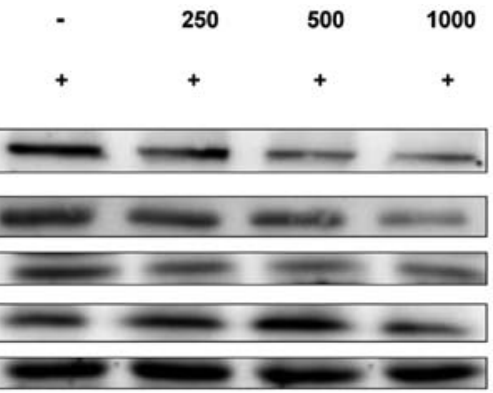

B

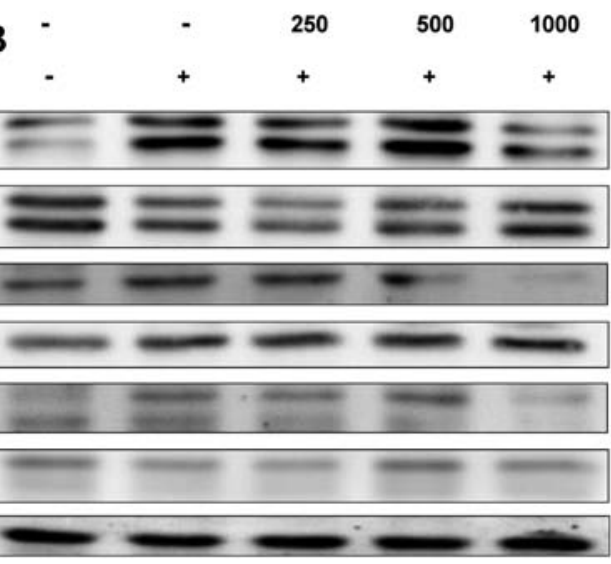

PPY1 (ng/ml)

LPS $(10 \mathrm{ng} / \mathrm{ml})$

iNOS

cox-2

TNF- $\alpha$

IL-1 $1 \beta$

GAPDH

PPY1 (ng/ml)

LPS $(10 \mathrm{ng} / \mathrm{ml})$

p-ERK

ERK

p-p38

p38

p-JNK

JNK

GAPDH

Figure 4. Effect of the bioactive peptide from P. yezoensis (PPY1) on the level of (A) pro-inflammatory mediators and cytokines, and (B) MAPK signaling in LPS-stimulated RAW 264.7 macrophages. RAW 264.7 cells were stimulated with LPS $(10 \mathrm{ng} / \mathrm{ml})$ and treated with PPY1 $(250,500$ and $1,000 \mathrm{ng} / \mathrm{ml})$. The whole cell sample ( $35 \mu \mathrm{g}$ ) was loaded in SDS-PAGE and transferred to a PVDF membrane. The membrane was prepared and analyzed by immunoblotting using pro-inflammatory mediators (anti-iNOS, anti-COX-2, anti-TNF- $\alpha$ and anti-IL-1 $\beta$ ) and MAPKs (anti-phospho ERK, anti-ERK, anti-phospho p38, anti-p38, anti-phospho JNK and anti-JNK). GAPDH was the loading control for immunoblotting. MAPK, mitogen-activated protein kinase; LPS, lipopolysaccharide; iNOS, inducible nitric oxide synthase; COX-2, cyclooxygenase-2; TNF- $\alpha$, tumor necrosis factor- $\alpha$; IL, interleukin; ERK, extracellular signal-regulated kinase; JNK, c-jun $\mathrm{NH}_{2}$-terminal kinase.

increased NO production compared with the control group, whereas PPY1 co-treatment significantly $(\mathrm{P}<0.05)$ suppressed NO production in a dose-dependent manner (Fig. 3A).

Treatment of cells with 1,000 ng/ml of PPY1 inhibited NO release by $66.67 \%$. NO induction can be directly correlated with iNOS expression and other pro-inflammatory cytokines. The latter can be considered a molecular target for therapeutic compounds with anti-inflammatory action.

Whether PPY1 inhibited ROS generation in LPS-stimulated RAW 264.7 cells using CM-H2DCFDA was also examined, which has been widely used to study oxidative stress in macrophages (29). The fluorescence intensity corresponding to ROS produced by $10 \mathrm{ng} / \mathrm{ml}$ LPS-stimulated cells significantly shifted compared with that of non-stimulated cells. The ROS level was increased in LPS-stimulated cells (Fig. 3B). However, this increase was significantly reduced by $52.9 \%$ when the cells were treated with PPY1. These results suggested that PPY1 is effective in suppressing the production of ROS in macrophages.

Anti-inflammatory effects of PPY1. The expression of inflammatory enzymes iNOS and COX-2 was strongly induced in LPS-stimulated Raw 264.7 cells. The effects of PPY1 on iNOS and COX-2 expression were examined by western blot analysis. The overexpression in LPS-stimulated RAW 264.7 cells was suppressed by PPY1 in a dose-dependent manner at both levels. As the quantities of iNOS protein correlated with NO accumulation, these results suggest that PPY1 inhibited NO production by reducing iNOS protein expression. Cytokines are also produced during inflammation, and these are indicative of inflammatory progression. In response to LPS, TNF- $\alpha$ and IL-1 $\beta$ were significantly upregulated. Treatment with PPY1 considerably inhibited the LPS induction of TNF- $\alpha$ and IL-1 $\beta$ in a dose-dependent way (Fig. 4).

Effects of PPY1 on MAPK signaling. As MAPK signaling has a critical role in the response of cells to various cytokines and stresses, the effects of PPY1 were investigated on the MAPK pathways. The phosphorylation of ERK, p38 and JNK was induced by LPS stimulation. Co-treatment of PPY1 significantly reduced the level of p-ERK1/2, p-JNK, and p-p38 levels in LPS-stimulated RAW 264.7 cells (Fig. 4). The results show that inhibition of pro-inflammatory mediator expression by 
PPY1 in LPS-stimulated RAW 264.7 cells was associated with downregulation of ERK, p38, and JNK phosphorylation.

\section{Discussion}

There is increasing interest in the natural products obtained from marine resources, which are regarded as the last remaining reservoir for materials to meet future therapeutic requirements (30). In the present study, the bioactive peptide (PPY1) from the marine algae, $P$. yezoensis, was investigated for the presence of a potent anti-inflammatory agent. $P$. yezoensis is the diverse group of photosynthetic marine organisms that has adapted to surviving in highly complex and competitive environments, including severe salinity levels, temperature variations, low light intensities, and low and high tides (31). Therefore, it is assumed that specific peptides from $P$. yezoensis can serve in prominent biological activities, particularly those involving anti-inflammatory effects. Our previous studies demonstrated the effects of a peptide from $P$. yezoensis on cell proliferation and the associated signaling pathway in IEC-6 and MCF-7 cells (32-35). Our study was inconsistent with the general finding that short peptides with 2-10 amino acids exert more bioactive properties than their parent native proteins or large polypeptides. The present study developed PPY1 as a bioactive peptide. PPY1 was composed of five amino acids (K-A-Q-A-D) by enzymatic hydrolysis from P. yezoensisa. Anti-inflammatory activity, by suppression of inflammatory cytokines, was observed. The result was similar to the study reporting that the function of any peptide is mostly dependent on its specific amino acid composition (36), and these amino acids containing His, Ala, and Lys have been shown to have strong activity against intracellular NO and ROS production (37). The present results also suggest that PPY1 may contribute to preventing inflammation through inhibition of the expression of the inflammatory mediators, iNOS, COX-2, in LPS-stimulated RAW 264.7 macrophages without having any effect on cell viability. TNF- $\alpha$ and IL-1 $\beta$ have been described as important inflammatory cytokines, with upregulation linked to the pathogenesis of numerous infectious and inflammatory diseases, including cancer (38). MAPKs regulate various inflammatory and immune responses, including LPS-induced expression of COX-2 and iNOS in macrophages. The inhibition of MAPK family members, ERK, p38 and JNK, blocks the production of pro-inflammatory cytokines, which resulted from the suppression of the phosphorylation of these MAPK members.

Therefore, the results of the present study have promising implications for biomedical research and warrant further in vivo studies. Future study should verify the role of these bioactive peptides in the prevention and treatment of other inflammatory-related disorders, such as degenerative diseases and aging.

\section{Acknowledgements}

The present study was supported by the Basic Science Research Program through the National Research Foundation of Korea funded by the Ministry of Education (grant no. 2012R1A6A1028677).

\section{References}

1. Niwa K: Genetic analysis of artificial green and red mutants of Porphyra yezoensis Ueda (Bangiales, Rhodophyta). Aquaculture 308: 6-12, 2012.

2. Wang Y, Cai C, Li B, Liu C and He P: Photodynamic effect of two kinds of phycobiliproteins on human liver cancer cell line SMMC-7721 in vitro. Sheng Wu Gong Cheng Xue Bao 25: 1417-1423, 2009 (In Chinese)

3. Kim S, You DH, Han T and Choi EM: Modulation of viability and apoptosis of UVB-exposed human keratinocyte HaCaT cells by aqueous methanol extract of laver (Porphyra yezoensis). J Photochem Photobiol B 141: 301-307, 2014.

4. Ryu J, Park SJ, Kim IH, Choi YH and Nam TJ: Protective effect of porphyra-334 on UVA-induced photoaging in human skin fibroblasts. Int J Mol Med 34: 796-803, 2014.

5. Cian RE, Caballero MS, Sabbag N, González RJ and Drago SR: Bio-accessibility of bioactive compounds (ACE inhibitors and antioxidants) from extruded maize products added with a red seaweed Porphyra columbina. LWT-Food Sci Technol 55: 51-58, 2014.

6. Nakano T, Watanabe M, Sato M, Sato M and Takeuchi M: Characterization of catalase from the seaweed Porphyra yezoensis. Plant Sci 104: 127-133, 1995.

7. Cian RE, Martínez-Augustin O and Drago SR: Bioactive properties of peptides obtained by enzymatic hydrolysis from protein by products of Porphyra columbina. Food Res Int 49: 364-372, 2012.

8. Qu W, Ma H, Pan Z, Luo L, Wang Z and He R: Preparation and antihypertensive activity of peptides from Porphyra yezoensis. Food Chem 123: 14-20, 2010.

9. Jiang Z, Hama Y, Yamaguchi K and Oda T: Inhibitory effect of sulphated polysaccharide porphyran on nitric oxide production in lipopolysaccharide-stimulated RAW264.7 macrophages. J Biochem 151: 65-74, 2012.

10. Shin ES, Hwang HJ, Kim IH and Nam TJ: A glycoprotein from Porphyra yezoensis produces anti-inflammatory effects in liposaccharide-stimulated macrophages via the TLR4 signaling pathway. Int J Mol Med 28: 809-815, 2011.

11. Toyosaki T and Iwabuchi M: New antioxidant protein in seaweed (Porphyra yezoensis Ueda). Int J Food Sci Nutr 60 (Suppl 2): 46-56, 2009.

12. Choi JW, Kim YM, Park SJ, Kim IH and Nam TJ: Protective effect of Porphyra yezoensis glycoprotein on D-galactosamine induced cytotoxicity in Hepa 1clc7 cells. Mol Med Rep 11: 3914-3919, 2015.

13. Isaka $S$, Cho K, Nakazono S, Abu R, Ueno M, Kim D and Oda T: Antioxidant and anti-inflammatory activities of porphyran isolated from discolored nori (Porphyra yezoensis). Int J Biol Macromol 74: 68-75, 2015.

14. Eitsuka T, Nakagawa K, Igarashi M and Miyazawa T: Telomerase inhibition by sulfoquinovosyldiacylglycerol from edible purple laver (Porphyra yezoensis). Cancer Lett 212: 15-20, 2004.

15. Li L, Saga N and Mikami K: Effects of cell wall synthesis on cell polarity in the red alga Porphyra yezoensis. Plant Signal Behav 3: 1126-1128, 2008.

16. Hwang HJ, Kwon MJ, Kim IH and Nam TJ: Chemoprotective effects of a protein from the red algae Porphyra yezoensis on acetaminophen-induced liver injury in rats. Phytother Res 22: 1149-1153, 2008.

17. Guo TT, Xu HL, Zhang LX, Zhang JP, Guo YF, Gu JW and He PM: In vivo protective effect of Porphyra yezoensis polysaccharide against carbon tetrachloride induced hepatotoxicity in mice. Regul Toxicol Pharmacol 49: 101-106, 2007.

18. Vo TS, Ryu B and Kim SK: Purification of novel anti-inflammatory peptides from enzymatic hydrolysate of the edible microalgal Spirulina maxima. J Funct Foods 5: 1336-1346, 2013.

19. Mohamed S, Hashim SN and Rahman HA: Seaweeds: A sustainable functional food for complementary and alternative therapy. Trends Food Sci Technol 23: 83-96, 2012.

20. Kim SK and Wijesekara I: Development and biological activities of marine-derived bioactive peptides. J Funct Foods 2: 1-9, 2010.

21. Millán-Linares MC, Bermúdez B, Yust MM, Millan F and Pedroche J: Anti-inflammatory activity of lupine (Lupinus angustifolius L.) protein hydrolysates in THP-1-derived macrophages. J Funct Foods 8: 224-233, 2014.

22. Hernández-Ledesma B, Hsieh CC and de Lumen BO: Antioxidant and anti-inflammatory properties of cancer preventive peptide lunasin in RAW 264.7 macrophages. Biochem Biophys Res Commun 390: 803-808, 2009. 
23. Liew FY: The role of innate cytokines in inflammatory response. Immunol Lett 85: 131-134, 2003.

24. Baeuerle PA and Baltimore D: NF-к B: Ten years after. Cell 87: 13-20, 1996.

25. Kaminska B: MAPK signalling pathways as molecular targets for anti-inflammatory therapy - from molecular mechanisms to therapeutic benefits. Biochim Biophys Acta 1754: 253-262, 2005.

26. Zhu Y, Duan Z, Mo G, Shen C, Lv L, Chen W and Lai R: A novel 26RFa peptide containing both analgesic and anti-inflammatory functions from Chinese tree shrew. Biochimie 102: 112-116, 2014.

27. Lee M, Kovacs-Nolan J, Archbold T, Fan MZ, Juneja LR, Okubo T and Mine Y: Therapeutic potential of hen egg white peptides for the treatment of intestinal inflammation. J Funct Foods 1: 161-169, 2009.

28. Green LC, Wagner DA, Glogowski J, Skipper PL, Wishnok JS and Tannenbaum SR: Analysis of nitrate, nitrite, and $\left.{ }^{[5} \mathrm{N}\right]$ nitrate in biological fluids. Anal Biochem 126: 131-138, 1982.

29. Xu MZ, Lee WS, Han JM, Oh HW, Park DS, Tian GR, Jeong TS and Park HY: Antioxidant and anti-inflammatory activities of $\mathrm{N}$-acetyldopamine dimers from Periostracum Cicadae. Bioorg Med Chem 14: 7826-7834, 2006.

30. Samarakoon K and Jeon YJ: Bio-functionalities of proteins derived from marine algae. Food Res Int 48: 948-960, 2012.

31. Plaza M, Cifuentes A and Ibáñez E: In the search of new functional food ingredients from algae. Trends Food Sci Technol 19: 31-39, 2008.
32. Lee MK, Kim IH, Choi YH and Nam TJ: A peptide from Porphyra yezoensis stimulates the proliferation of IEC- 6 cells by activating the insulin-like growth factor I receptor signaling pathway. Int J Mol Med 35: 533-538, 2015.

33. Lee MK, Kim IH, Choi YH, Choi JW, Kim YM and Nam TJ: The proliferative effects of Pyropia yezoensis peptide on IEC-6 cells are mediated through the epidermal growth factor receptor signaling pathway. Int J Mol Med 35: 909-914, 2015.

34. Park SJ, Ryu J, Kim IH, Choi YH and Nam TJ: Induction of apoptosis by a peptide from Porphyra yezoensis: Regulation of the insulin-like growth factor I receptor signaling pathway in MCF-7 cells. Int J Oncol 45: 1011-1016, 2014.

35. Park SJ, Ryu J, Kim IH, Choi YH and Nam TJ: Activation of the mTOR signaling pathway in breast cancer MCF 7 cells by a peptide derived from Porphyra yezoensis. Oncol Rep 33: 19-24, 2015.

36. Wang B, Li L, Chi CF, Ma JH, Luo HY and Xu YF: Purification and characterisation of a novel antioxidant peptide derived from blue mussel (Mytilus edulis) protein hydrolysate. Food Chem 138: 1713-1719, 2013.

37. He R, Girgih AT, Malomo SA, Ju X and Aluko RE: Antioxidant activities of enzymatic rapeseed protein hydrolysates and the membrane ultrafiltration fractions. J Funct Foods 5: 219-227, 2013.

38. Xie C, Kang J, Li Z, Schauss AG, Badger TM, Nagarajan S, Wu T and $\mathrm{Wu} \mathrm{X}$ : The açaí flavonoid velutin is a potent anti-inflammatory agent: Blockade of LPS-mediated TNF- $\alpha$ and IL-6 production through inhibiting NF- $\mathrm{B}$ activation and MAPK pathway. J Nutr Biochem 23: 1184-1191, 2012. 